\title{
A Mixed Problem with an Integral Two-Space-Variables Condition for Parabolic Equation with The Bessel Operator
}

\author{
Bouziani Abdelfatah, Oussaeif Taki-Eddine, and Ben Aoua Leila \\ Department of Mathematics and Informatics, The Larbi Ben M'hidi University, 04000 Oum El Bouaghi, Algeria \\ Correspondence should be addressed to Oussaeif Taki-Eddine; taki_maths@live.fr
}

Received 27 October 2012; Revised 12 March 2013; Accepted 29 March 2013

Academic Editor: Mohsen Tadi

Copyright (c) 2013 Bouziani Abdelfatah et al. This is an open access article distributed under the Creative Commons Attribution License, which permits unrestricted use, distribution, and reproduction in any medium, provided the original work is properly cited.

We study a mixed problem with an integral two-space-variables condition for parabolic equation with the Bessel operator. The existence and uniqueness of the solution in functional weighted Sobolev space are proved. The proof is based on a priori estimate "energy inequality" and the density of the range of the operator generated by the problem considered.

\section{Introduction}

The importance of boundary value problems with integral boundary condition has been pointed out by Samarskiı [1] and problems with integral conditions for parabolic equations were treated by Kamynin [2], Ionkin [3], Yurchuk [4], Benouar and Yurchuk [5], Bouziani [6], Bouziani and Benouar [7, 8], and Mesloub and Bouziani [9]. Other parabolic problems also arise in plasma physics by Samarskiu [1], heat conduction by Cannon [10], Ionkin [3], dynamics of ground waters by Nakhushev [11], Vodakhova [12], Kartynnik [13], and Lin [14]. Regular case of this problem is studied in [15]. The problem where the equation contains an operator of the form $(\partial / \partial x)(a(x, t)(\partial u / \partial x))$, instead of Bessel operator, is treated in [16]. Similar problems for second-order parabolic equations are investegated by the potential method in [17]. Later problems with an integral two-space-variables condition for parabolic equations were treated by Marhoune [18], Marhoune and Lakhal [19]. Motivated by this, we study a mixed problem with an integral two-space-variables condition for parabolic equation with the Bessel operator.

\section{Setting of the Problem}

In the rectangular domain $\Omega=(0,1) \times(0, T)$, with $T<\infty$, we consider the following equation:

$$
\mathscr{L} u=\frac{\partial u}{\partial t}-\left(\frac{\partial^{2} u}{\partial x^{2}}+\frac{1}{x} \frac{\partial u}{\partial x}\right)=f(x, t),
$$

with the initial data

$$
\ell u=u(x, 0)=\varphi(x), \quad x \in(0,1),
$$

Neumann boundary condition

$$
\frac{\partial u}{\partial x}(1, t)=0
$$

and the integral condition

$$
\begin{aligned}
& \int_{0}^{\alpha} x u(x, t) d x+\int_{\beta}^{1} x u(x, t) d x=0, \\
& \beta>\alpha>0, \quad \alpha+\beta=1, \quad t \in(0, T),
\end{aligned}
$$

where $\varphi$ is a known function.

We shall assume that the functions $\varphi$ and $f$ satisfy the compatibility condition with (4), that is,

$$
\begin{gathered}
\int_{0}^{\alpha} x \varphi(x) d x+\int_{\beta}^{1} x \varphi(x) d x=0, \\
\int_{0}^{\alpha} x f(x, t) d x+\int_{\beta}^{1} x f(x, t) d x=0 .
\end{gathered}
$$


The presence of integral terms in boundary condition can, in general, greatly complicate the application of standard functional or numerical techniques, specially the integral two-space-variables condition. Then to avoid this difficulty, we introduce a technique for transfering this problem to another classically less complicated one that does not contain integral conditions. For that, we establish the following lemma.

Lemma 1. Problem (1)-(4) is equivalent to the following problem $(P R)$ :

$$
\begin{gathered}
\mathscr{L} u=\frac{\partial u}{\partial t}-\left(\frac{\partial^{2} u}{\partial x^{2}}+\frac{1}{x} \frac{\partial u}{\partial x}\right)=f(x, t), \\
\ell u=u(x, 0)=\varphi(x), \quad x \in(0,1), \\
\alpha \frac{\partial u}{\partial x}(\alpha, t)=\beta \frac{\partial u}{\partial x}(\beta, t), \quad \frac{\partial u}{\partial x}(1, t)=0 .
\end{gathered}
$$

Proof. Let $u(x, t)$ be a solution of (1)-(4), we prove that

$$
\frac{\partial u}{\partial x}(\alpha, t)=\frac{\partial u}{\partial x}(\beta, t)
$$

So, multiplying (1) by $x$ and integrating with respect to $x$ over $(0, \alpha)$ and $(\beta, 1)$ and taking into account (4) and (6), we obtain

$$
\begin{gathered}
\left.x \frac{\partial u}{\partial x}\right|_{x=0} ^{x=\alpha} d x+\left.x \frac{\partial u}{\partial x}\right|_{x=\beta} ^{x=1} d x=0, \quad \text { this implies } \\
\alpha \frac{\partial u}{\partial x}(\alpha, t)+\frac{\partial u}{\partial x}(1, t)-\beta \frac{\partial u}{\partial x}(\beta, t)=0 .
\end{gathered}
$$

Then, from (3), we obtain

$$
\alpha \frac{\partial u}{\partial x}(\alpha, t)=\beta \frac{\partial u}{\partial x}(\beta, t)
$$

Let now $u(x, t)$ be a solution of (PR), we are bound to prove that

$$
\int_{0}^{\alpha} x u(x, t) d x+\int_{\beta}^{1} x u(x, t) d x=0 .
$$

So, multiplying (1) by $x$ and integrating with respect to $x$ over $(0, \alpha)$ and $(\beta, 1)$ and taking into account

$$
\alpha \frac{\partial u}{\partial x}(\alpha, t)=\beta \frac{\partial u}{\partial x}(\beta, t), \quad \frac{\partial u}{\partial x}(1, t)=0,
$$

we obtain

$$
\begin{aligned}
& \frac{\partial}{\partial t} \int_{0}^{\alpha} x u(x, t) d x=\int_{0}^{\alpha} x f(x, t) d x, \\
& \frac{\partial}{\partial t} \int_{\beta}^{1} x u(x, t) d x=\int_{\beta}^{1} x f(x, t) d x,
\end{aligned}
$$

combining the two preceding equations, and from (6) we get

$$
\int_{0}^{\alpha} x u(x, t) d x+\int_{\beta}^{1} x u(x, t) d x=0 .
$$

\section{A Priori Estimate}

The method used here is one of the most efficient functional analysis methods in solving partial differential equations with integral conditions, the so-called a priori estimate method or the energy-integral method. This method is essentially based on the construction of multiplicators for each specific given problem, which provides the a priori estimate from which it is possible to establish the solvability of the posed problem. More precisely, the proof is based on an energy inequality and the density of the range of the operator generated by the abstract formulation of the stated problem. But here we use the energy inequality method for the equivalent problem (PR) given in Lemma 1; so to investigate the posed problem, we introduce the needed function spaces.

We introduce function spaces needed in our investigation. We denote by $L_{\rho}^{2}(\Omega)$ the weighted Lebesgue space that consists of all measurable functions $u$ equipped with the finite norm

$$
\|u\|_{L_{\rho}^{2}(\Omega)}=\left(\int_{\Omega} \rho(x)|u(x, \cdot)|^{2} d x\right)^{1 / 2},
$$

where $\rho(x)=\left(x^{2}+x\right)$. If $\rho(x)=1, L_{\rho}^{2}(\Omega)$ are identified with the standard spaces $L^{2}(\Omega)$.

In this paper, we prove the existence and the uniqueness for solution of the problem (1)-(4) as a solution of the operator equation

$$
L u=\mathscr{F},
$$

where $L=(\mathscr{L}, \ell)$, with domain of definition $B$ consisting of functions $u \in L_{\rho}^{2}(\Omega)$ such that $\partial u / \partial t, \partial u / \partial x, \partial^{2} u / \partial x^{2}$, $\partial^{2} u / \partial t \partial x \in L_{\rho}^{2}(\Omega)$, and $u$ satisfy condition (4); the operator $L$ is considered from $B$ to $F$, where $B$ is the Banach space consisting of all functions $u(x, t)$ having a finite norm

$$
\begin{aligned}
\|u\|_{B}^{2}= & \int_{\Omega}\left(x^{2}+x\right)\left(\frac{\partial u}{\partial t}\right)^{2} d x d t \\
& +\sup _{0 \leq \tau \leq T} \int_{0}^{1}\left(x^{2}+x\right)\left(\frac{\partial u(x, \tau)}{\partial x}\right)^{2} d x
\end{aligned}
$$

and $F$ is the Hilbert space consisting of all elements $\mathscr{F}=$ $(f, \varphi)$ for which the norm

$$
\|\mathscr{F}\|_{F}^{2}=\int_{\Omega} f^{2} d x d t+\int_{0}^{1}\left(x^{2}+x\right)\left(\frac{\partial \varphi}{\partial x}\right)^{2} d x
$$

is finite.

Theorem 2. For any function $u \in B$, one has the estimate

$$
\|u\|_{B} \leq c\|L u\|_{F}
$$

where $c$ is a positive constant independent of $u$. 
Proof. Multiplying (1) by the following $M u$ :

$$
M u= \begin{cases}\left(x^{2}-x\right) \frac{\partial u}{\partial x}+\left(x^{2}+x\right) \frac{\partial u}{\partial t}, & 0 \leq x \leq \alpha, \\ -x \frac{\partial u}{\partial x}+\left(x^{2}+x\right) \frac{\partial u}{\partial t}, & \alpha \leq x \leq \beta, \\ \left(x^{2}-x\right) \frac{\partial u}{\partial x}+\left(x^{2}+x\right) \frac{\partial u}{\partial t}, & \beta \leq x \leq 1,\end{cases}
$$

and integrating over $\Omega^{\tau}$, where $\Omega^{\tau}=(0,1) \times(0, \tau)$,

$$
\begin{aligned}
& \int_{\Omega^{\tau}} \mathscr{L} u \cdot M u d x d t \\
& \iint_{\Omega_{\alpha}^{\tau}}\left(x^{2}-x\right) \frac{\partial u}{\partial x} \frac{\partial u}{\partial t} d x d t \\
& +\int_{\Omega_{\alpha}^{\tau}}(1-x)\left(\frac{\partial u}{\partial x}\right)^{2} d x d t \\
& +\int_{\Omega_{\alpha}^{\tau}}^{c}\left(x^{2}+x\right)\left(\frac{\partial u}{\partial t}\right)^{2} d x d t \\
& -\int_{\Omega_{\alpha}^{\tau}}^{\alpha_{\alpha}}\left(x-x^{2}\right) \frac{\partial u}{\partial x} \frac{\partial^{2} u}{\partial x^{2}} d x d t \\
& -\int_{\Omega_{\alpha}^{\tau}}^{\Omega_{\alpha}}(x+1) \frac{\partial}{\partial x}\left(x \frac{\partial u}{\partial x}\right) \frac{\partial u}{\partial t} d x d t \\
& =\int_{\Omega_{\alpha}^{\tau}} f \cdot\left(\left(x^{2}-x\right) \frac{\partial u}{\partial x}\right. \\
& \left.+\left(x^{2}+x\right) \frac{\partial u}{\partial t}\right) d x d t, \quad \Omega_{\alpha}^{\tau}, \\
& \int_{\Omega_{\alpha, \beta}^{\tau}}\left(x^{2}+x\right)\left(\frac{\partial u}{\partial t}\right)^{2} d x d t \\
& -\int_{\Omega_{\alpha, \beta}^{\tau}} x \frac{\partial u}{\partial x} \frac{\partial u}{\partial t} d x d t+\int_{\Omega_{\alpha, \beta}^{\tau}}\left(\frac{\partial u}{\partial x}\right)^{2} d x d t \\
& +\int_{\Omega_{\alpha, \beta}^{\tau}}^{\alpha x} x \frac{\partial u}{\partial x} \frac{\partial^{2} u}{\partial x^{2}} d x d t \\
& -\int_{\Omega_{\alpha, \beta}^{\tau}}^{\alpha, \beta}(x+1) \frac{\partial}{\partial x}\left(x \frac{\partial u}{\partial x}\right) \frac{\partial u}{\partial t} d x d t \\
& =\int_{\Omega_{\alpha, \beta}^{\tau}} f \cdot\left(-x \frac{\partial u}{\partial x}\right. \\
& \left.+\left(x^{2}+x\right) \frac{\partial u}{\partial t}\right) d x d t, \quad \Omega_{\alpha, \beta}^{\tau}, \\
& \int_{\Omega_{\beta}^{\tau}}\left(x^{2}+x\right)\left(\frac{\partial u}{\partial t}\right)^{2} d x d t \\
& +\int_{\Omega_{\beta}^{\tau}}\left(x^{2}-x\right) \frac{\partial u}{\partial x} \frac{\partial u}{\partial t} d x d t \\
& +\int_{\Omega_{\beta}^{\tau}}(1-x)\left(\frac{\partial u}{\partial x}\right)^{2} d x d t \\
& +\int_{\Omega_{\beta}^{\tau}}\left(x-x^{2}\right) \frac{\partial u}{\partial x} \frac{\partial^{2} u}{\partial x^{2}} d x d t \\
& -\int_{\Omega_{\beta}^{\tau}}^{\beta}(x+1) \frac{\partial}{\partial x}\left(x \frac{\partial u}{\partial x}\right) \frac{\partial u}{\partial t} d x d t \\
& =\int_{\Omega_{\beta}^{\tau}} f \cdot\left(\left(x^{2}-x\right) \frac{\partial u}{\partial x}\right. \\
& \left.+\left(x^{2}+x\right) \frac{\partial u}{\partial t}\right) d x d t, \quad \Omega_{\beta}^{\tau} .
\end{aligned}
$$

Integration by parts, we obtain

$$
\begin{aligned}
-\int_{\Omega_{\alpha}^{\tau}} & \left(x-x^{2}\right) \frac{\partial u}{\partial x} \frac{\partial^{2} u}{\partial x^{2}} d x d t \\
= & \int_{0}^{\tau}\left(\frac{\alpha}{2}-\frac{\alpha^{2}}{2}\right)\left(\frac{\partial u}{\partial x}(\alpha, t)\right)^{2} d t \\
& -\int_{\Omega_{\alpha}^{\tau}}\left(\frac{1}{2}-x\right)\left(\frac{\partial u}{\partial x}\right)^{2} d x d t \\
-\int_{\Omega_{\alpha}^{\tau}}( & +1) \frac{\partial}{\partial x}\left(x \frac{\partial u}{\partial x}\right) \frac{\partial u}{\partial t} d x d t \\
= & -\int_{0}^{\tau}\left(\alpha^{2}+\alpha\right) \frac{\partial u}{\partial x}(\alpha, t) \frac{\partial u}{\partial t}(\alpha, t) d t \\
& +\int_{\Omega_{\alpha}^{\tau}} x \frac{\partial u}{\partial x} \frac{\partial u}{\partial t} d x d t+\frac{1}{2} \int_{0}^{\alpha}\left(x^{2}+x\right)\left(\frac{\partial u(\cdot, \tau)}{\partial x}\right)^{2} d x \\
& -\frac{1}{2} \int_{0}^{\alpha}\left(x^{2}+x\right)\left(\frac{\partial \varphi}{\partial x}\right)^{2} d x,
\end{aligned}
$$$$
\int_{\Omega_{\alpha, \beta}^{\tau}} x \frac{\partial u}{\partial x} \frac{\partial^{2} u}{\partial x^{2}} d x d t
$$$$
=\frac{\beta}{2} \int_{0}^{\tau}\left(\frac{\partial u}{\partial x}(\beta, t)\right)^{2} d t-\frac{\alpha}{2} \int_{0}^{\tau}\left(\frac{\partial u}{\partial x}(\alpha, t)\right)^{2} d t
$$$$
-\frac{1}{2} \int_{\Omega_{\alpha, \beta}^{\tau}}\left(\frac{\partial u}{\partial x}\right)^{2} d x d t,
$$$$
-\int_{\Omega_{\alpha, \beta}^{\tau}}(x+1) \frac{\partial}{\partial x}\left(x \frac{\partial u}{\partial x}\right) \frac{\partial u}{\partial t} d x d t
$$$$
=-\int_{0}^{\tau}\left(\beta^{2}+\beta\right) \frac{\partial u}{\partial x}(\beta, t) \frac{\partial u}{\partial t}(\beta, t) d t
$$$$
+\int_{0}^{\tau}\left(\alpha^{2}+\alpha\right) \frac{\partial u}{\partial x}(\alpha, t) \frac{\partial u}{\partial t}(\alpha, t) d t
$$$$
+\int_{\Omega_{\alpha, \beta}} x \frac{\partial u}{\partial x} \frac{\partial u}{\partial t} d x d t
$$$$
+\frac{1}{2} \int_{\alpha}^{\beta}\left(x^{2}+x\right)\left(\frac{\partial u(\cdot, \tau)}{\partial x}\right)^{2} d x
$$$$
-\frac{1}{2} \int_{\alpha}^{\beta}\left(x^{2}+x\right)\left(\frac{\partial \varphi}{\partial x}\right)^{2} d x,
$$$$
\int_{\Omega_{\beta}^{\tau}}\left(x-x^{2}\right) \frac{\partial u}{\partial x} \frac{\partial^{2} u}{\partial x^{2}} d x d t
$$$$
=\int_{0}^{\tau}\left(\frac{\beta^{2}}{2}-\frac{\beta}{2}\right)\left(\frac{\partial u}{\partial x}(\beta, t)\right)^{2} d t
$$$$
-\int_{\Omega_{\beta}^{\tau}}\left(\frac{1}{2}-x\right)\left(\frac{\partial u}{\partial x}\right)^{2} d x d t,
$$ 


$$
\begin{aligned}
-\int_{\Omega_{\beta}^{\tau}} & (x+1) \frac{\partial}{\partial x}\left(x \frac{\partial u}{\partial x}\right) \frac{\partial u}{\partial t} d x d t \\
= & \int_{0}^{\tau}\left(\beta^{2}+\beta\right) \frac{\partial u}{\partial x}(\beta, t) \frac{\partial u}{\partial t}(\beta, t) d t \\
& +\int_{\Omega_{\beta}^{\tau}} x \frac{\partial u}{\partial x} \frac{\partial u}{\partial t} d x d t \\
& +\frac{1}{2} \int_{\beta}^{1}\left(x^{2}+x\right)\left(\frac{\partial u(\cdot, \tau)}{\partial x}\right)^{2} d x \\
& -\frac{1}{2} \int_{\beta}^{1}\left(x^{2}+x\right)\left(\frac{\partial \varphi}{\partial x}\right)^{2} d x
\end{aligned}
$$

and by using the Cauchy's $\varepsilon$-inequality, we get

$$
\begin{aligned}
& \int_{\Omega_{\alpha}^{\tau}} x^{2} \frac{\partial u}{\partial x} \frac{\partial u}{\partial t} d x d t \\
& \leq \frac{\varepsilon}{2} \int_{\Omega_{\alpha}^{\tau}} x^{2}\left(\frac{\partial u}{\partial x}\right)^{2} d x d t+\frac{1}{2 \varepsilon} \int_{\Omega_{\alpha}^{\tau}} x^{2}\left(\frac{\partial u}{\partial t}\right)^{2} d x d t \\
& \leq \frac{1}{4} \int_{\Omega_{\alpha}^{\tau}}\left(\frac{\partial u}{\partial x}\right)^{2} d x d t \\
& +\frac{1}{4\left(\alpha^{2}+\alpha\right)} \int_{\Omega_{\alpha}^{\tau}}\left(x^{2}+x\right)\left(\frac{\partial u}{\partial x}\right)^{2} d x d t \\
& +\frac{\left(\alpha^{2}+\alpha\right)}{2} \int_{\Omega_{\alpha}^{\tau}}\left(x^{2}+x\right)\left(\frac{\partial u}{\partial t}\right)^{2} d x d t \\
& \int_{\Omega_{\alpha}^{\tau}} f \cdot\left(\left(x^{2}-x\right) \frac{\partial u}{\partial x}+\left(x^{2}+x\right) \frac{\partial u}{\partial t}\right) d x d t \\
& \leq\left(\alpha^{2}+\alpha\right)^{2} \int_{\Omega_{\alpha}^{\tau}} f^{2} d x d t+\frac{1}{4} \int_{\Omega_{\alpha}^{\tau}}\left(\frac{\partial u}{\partial x}\right)^{2} d x d t \\
& +\frac{1}{4\left(\alpha^{2}+\alpha\right)} \int_{\Omega_{\alpha}^{\tau}}\left(x^{2}+x\right)\left(\frac{\partial u}{\partial x}\right)^{2} d x d t \\
& +\frac{1}{2\left(\alpha^{2}+\alpha\right)} \int_{\Omega_{\alpha}^{\tau}}\left(x^{2}+x\right)\left(\frac{\partial u}{\partial t}\right)^{2} d x d t, \\
& \int_{\Omega_{\alpha, \beta}^{\tau}} f \cdot\left(-x \frac{\partial u}{\partial x}+\left(x^{2}+x\right) \frac{\partial u}{\partial t}\right) d x d t \\
& \leq\left(\frac{(\beta-\alpha)\left(\left(\beta^{2}-\alpha^{2}\right)+(\beta-\alpha)\right)}{2}+\frac{1}{2}\right) \\
& \times \int_{\Omega_{\alpha, \beta}^{\tau}} f^{2} d x d t+\frac{1}{4} \int_{\Omega_{\alpha, \beta}^{\tau}}\left(\frac{\partial u}{\partial x}\right)^{2} d x d t \\
& +\frac{1}{4\left(\left(\beta^{2}-\alpha^{2}\right)+(\beta-\alpha)\right)}
\end{aligned}
$$

$$
\begin{gathered}
\times \int_{\Omega_{\alpha, \beta}^{\tau}}\left(x^{2}+x\right)\left(\frac{\partial u}{\partial x}\right)^{2} d x d t \\
+\frac{1}{2} \int_{\Omega_{\alpha, \beta}^{\tau}}\left(x^{2}+x\right)\left(\frac{\partial u}{\partial t}\right)^{2} \\
-\int_{\Omega_{\beta}^{\tau}} x^{2} \frac{\partial u}{\partial x} \frac{\partial u}{\partial t} d x d t \\
\leq \frac{\varepsilon}{2} \int_{\Omega_{\beta}^{\tau}} x\left(\frac{\partial u}{\partial x}\right)^{2} d x d t+\frac{1}{2 \varepsilon} \int_{\Omega_{\beta}^{\tau}} x\left(\frac{\partial u}{\partial t}\right)^{2} d x d t \\
\leq \frac{\varepsilon(1-\beta)}{2} \int_{\Omega_{\beta}^{\tau}}\left(\frac{\partial u}{\partial x}\right)^{2} d x d t
\end{gathered}
$$$$
+\frac{1}{2 \varepsilon} \int_{\Omega_{\beta}^{\tau}}\left(x^{2}+x\right)\left(\frac{\partial u}{\partial t}\right)^{2} d x d t
$$$$
\leq \frac{1}{4} \int_{\Omega_{\beta}^{\tau}}\left(\frac{\partial u}{\partial x}\right)^{2} d x d t
$$$$
+(1-\beta) \int_{\Omega_{\beta}^{\tau}}\left(x^{2}+x\right)\left(\frac{\partial u}{\partial t}\right)^{2} d x d t
$$$$
\int_{\Omega_{\beta}^{\tau}} f \cdot\left(\left(x^{2}-x\right) \frac{\partial u}{\partial x}+\left(x^{2}+x\right) \frac{\partial u}{\partial t}\right) d x d t
$$$$
\leq\left((1-\beta)^{2}+(1-\beta)\right)^{2} \int_{\Omega_{\beta}^{\tau}} f^{2}+\frac{1}{4} \int_{\Omega_{\beta}^{\tau}}\left(\frac{\partial u}{\partial x}\right)^{2}
$$$$
+\frac{1}{4\left((1-\beta)^{2}+(1-\beta)\right)} \int_{\Omega_{\beta}^{\tau}}\left(x^{2}+x\right)\left(\frac{\partial u}{\partial x}\right)^{2}
$$$$
+\frac{1}{2\left((1-\beta)^{2}+(1-\beta)\right)} \int_{\Omega_{\beta}^{\tau}}\left(x^{2}+x\right)\left(\frac{\partial u}{\partial t}\right)^{2} \text {. }
$$

Substituting (21)-(22) into $\int_{\Omega^{\tau}} \mathscr{L} u \cdot M u d x d t$, we obtain

$$
\begin{gathered}
\left(1-\frac{\alpha^{2}+\alpha}{2}-\frac{1}{2\left(\alpha^{2}+\alpha\right)}\right) \int_{\Omega_{\alpha}^{\tau}}\left(x^{2}+x\right)\left(\frac{\partial u}{\partial t}\right)^{2} d x d t \\
\quad+\frac{1}{2} \int_{0}^{\alpha}\left(x^{2}+x\right)\left(\frac{\partial u(\cdot, \tau)}{\partial x}\right)^{2} d x \\
\int_{0}^{\tau}\left(\frac{\alpha}{2}-\frac{\alpha^{2}}{2}\right)\left(\frac{\partial u}{\partial x}(\alpha, t)\right)^{2} d t \\
\quad-\int_{0}^{\tau}\left(\alpha^{2}+\alpha\right) \frac{\partial u}{\partial x}(\alpha, t) \frac{\partial u}{\partial t}(\alpha, t) d t
\end{gathered}
$$




$$
\begin{aligned}
& \leq\left(\alpha^{2}+\alpha\right)^{2} \int_{\Omega_{\alpha}^{\tau}} f^{2} d x d t \\
& +\frac{1}{2} \int_{0}^{\alpha}\left(x^{2}+x\right)\left(\frac{\partial \varphi}{\partial x}\right)^{2} d x \\
& +\frac{1}{2\left(\alpha^{2}+\alpha\right)} \int_{\Omega_{\alpha}^{\tau}}\left(x^{2}+x\right)\left(\frac{\partial u}{\partial x}\right)^{2} d x d t, \quad \Omega_{\alpha}^{\tau}, \\
& \frac{1}{2} \int_{\Omega_{\alpha, \beta}^{\tau}}\left(x^{2}+x\right)\left(\frac{\partial u}{\partial t}\right)^{2} \\
& +\frac{1}{2} \int_{\alpha}^{\beta}\left(x^{2}+x\right)\left(\frac{\partial u(\cdot, \tau)}{\partial x}\right)^{2} d x \\
& -\int_{0}^{\tau}\left(\beta^{2}+\beta\right) \frac{\partial u}{\partial x}(\beta, t) \frac{\partial u}{\partial t}(\beta, t) d t \\
& +\int_{0}^{\tau}\left(\frac{\alpha}{2}-\frac{\alpha^{2}}{2}\right)\left(\frac{\partial u}{\partial x}(\alpha, t)\right)^{2} d t \\
& \frac{\beta}{2} \int_{0}^{\tau}\left(\frac{\partial u}{\partial x}(\beta, t)\right)^{2} d t-\frac{\alpha}{2} \int_{0}^{\tau}\left(\frac{\partial u}{\partial x}(\alpha, t)\right)^{2} d t \\
& \leq\left(\frac{(\beta-\alpha)^{2}(\beta+\alpha+1)}{2}+\frac{1}{2}\right) \int_{\Omega_{\alpha, \beta}^{\tau}} f^{2} d x d t \\
& +\frac{1}{2} \int_{\alpha}^{\beta}\left(x^{2}+x\right)\left(\frac{\partial \varphi}{\partial x}\right)^{2} d x \\
& +\frac{1}{4\left(\left(\beta^{2}-\alpha^{2}\right)+(\beta-\alpha)\right)} \\
& \times \int_{\Omega_{\alpha, \beta}^{\tau}}\left(x^{2}+x\right)\left(\frac{\partial u}{\partial x}\right)^{2} d x d t, \quad \Omega_{\alpha, \beta}^{\tau}, \\
& \left(\beta-\frac{1}{2\left((1-\beta)^{2}+(1-\beta)\right)}\right) \int_{\Omega_{\beta}^{\tau}}\left(x^{2}+x\right)\left(\frac{\partial u}{\partial t}\right)^{2} d x d t \\
& +\frac{1}{2} \int_{\beta}^{1}\left(x^{2}+x\right)\left(\frac{\partial u(\cdot, \tau)}{\partial x}\right)^{2} d x \\
& \int_{0}^{\tau}\left(\frac{\beta^{2}}{2}-\frac{\beta}{2}\right)\left(\frac{\partial u}{\partial x}(\beta, t)\right)^{2} d t \\
& +\int_{0}^{\tau}\left(\beta^{2}+\beta\right) \frac{\partial u}{\partial x}(\beta, t) \frac{\partial u}{\partial t}(\beta, t) d t \\
& \leq\left((1-\beta)^{2}+(1-\beta)\right)^{2} \int_{\Omega_{\beta}^{\tau}} f^{2} d x d t \\
& +\frac{1}{2} \int_{\beta}^{1}\left(x^{2}+x\right)\left(\frac{\partial \varphi}{\partial x}\right)^{2} d x \\
& +\frac{1}{4\left((1-\beta)^{2}+(1-\beta)\right)} \\
& \times \int_{\Omega_{\beta}^{\tau}}\left(x^{2}+x\right)\left(\frac{\partial u}{\partial x}\right)^{2} d x d t, \quad \Omega_{\beta}^{\tau} \text {. }
\end{aligned}
$$$$
\frac{1}{2} \int_{\Omega_{\alpha, \beta}^{\tau}}\left(x^{2}+x\right)\left(\frac{\partial u}{\partial t}\right)^{2}
$$$$
+\frac{1}{2} \int_{\alpha}^{\beta}\left(x^{2}+x\right)\left(\frac{\partial u(\cdot, \tau)}{\partial x}\right)^{2} d x
$$$$
\leq\left(\frac{(\beta-\alpha)^{2}(\beta+\alpha+1)}{2}+\frac{1}{2}\right) \int_{\Omega_{\alpha, \beta}^{\tau}} f^{2} d x d t
$$$$
+\frac{1}{2} \int_{\alpha}^{\beta}\left(x^{2}+x\right)\left(\frac{\partial \varphi}{\partial x}\right)^{2} d x
$$$$
+\frac{1}{4\left(\left(\beta^{2}-\alpha^{2}\right)+(\beta-\alpha)\right)}
$$$$
\times \int_{\Omega_{\alpha, \beta}^{\tau}}\left(x^{2}+x\right)\left(\frac{\partial u}{\partial x}\right)^{2} d x d t, \quad \Omega_{\alpha, \beta}^{\tau},
$$$$
\left(\beta-\frac{1}{2\left((1-\beta)^{2}+(1-\beta)\right)}\right) \int_{\Omega_{\beta}^{\tau}}\left(x^{2}+x\right)\left(\frac{\partial u}{\partial t}\right)^{2} d x d t
$$$$
+\frac{1}{2} \int_{\beta}^{1}\left(x^{2}+x\right)\left(\frac{\partial u(\cdot, \tau)}{\partial x}\right)^{2} d x
$$$$
\leq\left((1-\beta)^{2}+(1-\beta)\right)^{2} \int_{\Omega_{\beta}^{\tau}} f^{2} d x d t
$$$$
+\frac{1}{2} \int_{\beta}^{1}\left(x^{2}+x\right)\left(\frac{\partial \varphi}{\partial x}\right)^{2} d x
$$$$
+\frac{1}{4\left((1-\beta)^{2}+(1-\beta)\right)}
$$$$
\times \int_{\Omega_{\beta}^{\tau}}\left(x^{2}+x\right)\left(\frac{\partial u}{\partial x}\right)^{2} d x d t . \quad \Omega_{\beta}^{\tau} .
$$ 
Or in $\Omega^{\tau}$, by combining (26), (27), and (28), we get

$$
\begin{aligned}
& \min \left(1-\frac{\alpha^{2}+\alpha}{2}-\frac{1}{2\left(\alpha^{2}+\alpha\right)}, \frac{1}{2}\right. \\
& \left.\beta-\frac{1}{2\left((1-\beta)^{2}+(1-\beta)\right)}\right) \\
& \quad \times \int_{\Omega^{\tau}}\left(x^{2}+x\right)\left(\frac{\partial u}{\partial t}\right)^{2} d x d t \\
& +\frac{1}{2} \int_{0}^{1}\left(x^{2}+x\right)\left(\frac{\partial u(\cdot, \tau)}{\partial x}\right)^{2} d x \\
& \leq \max \left(\left(\alpha^{2}+\alpha\right)^{2},\left(\frac{(\beta-\alpha)^{2}(\beta+\alpha+1)}{2}+\frac{1}{2}\right)\right. \\
& \times \int_{\Omega^{\tau}}\left(x^{2}+x\right)\left(\frac{\partial u}{\partial x}\right)^{2} d x d t . \\
& \quad \times \int_{\Omega^{\tau}} f^{2} d x d t+\frac{1}{2} \int_{0}^{1}\left(x^{2}+x\right)\left(\frac{\partial \varphi}{\partial x}\right)^{2} d x \\
& +\max \left(\frac{1}{2\left(\alpha^{2}+\alpha\right)}, \frac{1}{4\left(\left(\beta^{2}-\alpha^{2}\right)+(\beta-\alpha)\right)},\right.
\end{aligned}
$$

Using Lemma 1 in [20], one has

$$
\begin{gathered}
\left(\int_{\Omega}\left(x^{2}+x\right)\left(\frac{\partial u}{\partial t}\right)^{2} d x d t+\int_{0}^{1}\left(x^{2}+x\right)\left(\frac{\partial u(\cdot, \tau)}{\partial x}\right)^{2} d x\right) \\
\leq k\left(\int_{\Omega} f^{2} d x d t+\int_{0}^{1}\left(x^{2}+x\right)\left(\frac{\partial \varphi}{\partial x}\right)^{2} d x\right)
\end{gathered}
$$

where

$$
\begin{aligned}
k= & \max \left(\left(\alpha^{2}+\alpha\right)^{2},\left(\frac{(\beta-\alpha)^{2}(\beta+\alpha+1)}{2}+\frac{1}{2}\right),\right. \\
& \times\left(\operatorname { m i n } \left(1-\frac{\alpha^{2}+\alpha}{2}-\frac{1}{2\left(\alpha^{2}+\alpha\right)}, \frac{1}{2},\right.\right. \\
& \left.\left.\left.\quad \beta-\frac{1}{2\left((1-\beta)^{2}+(1-\beta)\right)}\right)\right)^{2}\right) \\
& \times e^{\left(\max \left(1 / 2\left(\alpha^{2}+\alpha\right), 1 / 4\left(\left(\beta^{2}-\alpha^{2}\right)+(\beta-\alpha)\right), 1 / 4\left((1-\beta)^{2}+(1-\beta)\right)\right) T\right) .}
\end{aligned}
$$

The right-hand side of (30) is independent of $\tau$, hence replacing the left-hand side by its upper bound with respect to $\tau$ from 0 to $T$, we obtain the desired inequality, where $c=(k)^{1 / 2}$.

Corollary 3. A solution of the problem (1)-(4) is unique if it exists and depends continuously on $\mathscr{F} \in F$.

\section{Existence of Solution}

To show the existance of solutions, we prove that $R(L)$ is dense in $F$ for all $u \in B$ and for arbitrary $\mathscr{F}=(f, \varphi) \in F$.

Theorem 4. Suppose the conditions of Theorem 2 are satisfied. Then the problem (1)-(4) admits a unique solution $u=L^{-1} \mathscr{F}$.

Proof. First we prove that $R(L)$ is dense in $F$ for the special case where $D(L) \equiv B$ is reduced to $D_{0}(L)$, where $D_{0}(L)=$ $\{u, u \in D(L): \ell u=0\}$.

Proposition 5. Let the conditions of Theorem 4 be satisfied. if for $\omega \in L^{2}(\Omega)$ and for all $u \in D_{0}(L)$, one has

$$
\int_{\Omega} \mathscr{L} u \cdot \omega d x d t=0
$$

then $\omega$ vanishes almost everywhere in $\Omega$.

Proof. The scalar product of $F$ is defined by

$$
\begin{aligned}
(L u, \omega)_{F}= & \int_{\Omega} \mathscr{L} u \cdot \omega d x d t \\
& +\int_{0}^{1}\left(x^{2}+x\right)\left(\frac{\partial \ell u}{\partial x}\right)\left(\frac{\partial \omega_{0}}{\partial x}\right) d x .
\end{aligned}
$$

the equality (32) can be written as follows:

$$
\int_{\Omega} \frac{\partial u}{\partial t} \cdot \omega d x d t=\int_{\Omega} \frac{1}{x} \frac{\partial}{\partial x}\left(x \frac{\partial u}{\partial x}\right) \cdot \omega d x d t
$$

if we put

$$
u=\mathfrak{J}_{t}\left(e^{k t} z\right)=\int_{0}^{t} e^{k \tau} z(x, \tau) d \tau
$$

where $k$ is a strictly positive constant and $z, \partial z / \partial x$, $(\partial / \partial x)\left(\partial \mathfrak{I}_{t}\left(e^{k t} z\right) / \partial x\right) \in L^{2}(\Omega)$, then, $u$ satisfies the boundary conditions in (PR). As a result of (34), we obtain the equality

$$
\int_{\Omega} e^{k t} z \omega d x d t=\int_{\Omega} \frac{1}{x} \frac{\partial}{\partial x}\left(x \frac{\partial \mathfrak{I}_{t}\left(e^{k t} z\right)}{\partial x}\right) \cdot \omega d x d t
$$

The left-hand side of (36) shows that the mapping

$$
L^{2}(\Omega) \ni z \longrightarrow \int_{\Omega} \frac{1}{x} \frac{\partial}{\partial x}\left(x \frac{\partial \Im_{t}\left(e^{k t} z\right)}{\partial x}\right) \cdot \omega d x d t
$$


is a continuous linear functional of $z$. From the right-hand side of (36) there follows that is true if the function $\omega$ has the following properties:

$$
\begin{gathered}
\frac{1}{x} \frac{\partial \omega}{\partial x}, \frac{\partial \mathfrak{I}_{t}^{\star} \omega}{\partial x}, \frac{1}{x} \frac{\partial \mathfrak{\Im}_{t}^{\star} \omega}{\partial x}, \frac{\partial^{2} \mathfrak{\Im}_{t}^{\star} \omega}{\partial x^{2}}, \mathfrak{\Im}_{t}^{\star}\left(\frac{\omega}{x^{2}}\right) \in L^{2}(\Omega), \\
\frac{\partial \omega}{\partial x}(\alpha, 0)=\frac{\partial \omega}{\partial x}(\beta, 0)=\frac{\partial \omega}{\partial x}(1,0)=0, \quad \frac{\partial \omega}{\partial x}(1, t)=0 .
\end{gathered}
$$

In terms of the given function $\omega$ and from the equality (36), we give the function $\omega$ in terms of $z$ as follows:

$$
\omega= \begin{cases}-\left(x-\frac{x^{2}}{2}\right) \frac{\partial z}{\partial x}, & 0 \leq x \leq \alpha \\ -x \frac{\partial z}{\partial x}, & \alpha \leq x \leq \beta \\ -\left(x-\frac{x^{2}}{2}\right) \frac{\partial z}{\partial x}, & \beta \leq x \leq 1,\end{cases}
$$

and $z$ satisfies the same conditions of the function $u$ in (PR):

$$
\begin{gathered}
\alpha \frac{\partial z}{\partial x}(\alpha, t)=\beta \frac{\partial z}{\partial x}(\beta, t), \\
\frac{\partial z}{\partial x}(1, t)=0, \quad z(\alpha, t)=z(\beta, t)=0 .
\end{gathered}
$$

Replacing $\omega$ in (36) by its representation (39) and integrating by parts each term of (36) and by taking the conditions (40) and (41), we obtain

$$
\begin{aligned}
& \int_{\Omega^{\tau}} e^{k t} z \omega d x d t \\
& =\left\{\begin{array}{c}
-\frac{1}{2} \int_{0}^{\tau}\left(\alpha-\frac{\alpha^{2}}{2}\right) \Im_{t} e^{k t}\left(\frac{\partial z(\alpha, t)}{\partial x}\right)^{2} d x d t \\
-\frac{1}{2} \int_{\Omega_{\alpha}^{\tau}}(1-x) \Im_{t} e^{k t}\left(\frac{\partial z}{\partial x}\right)^{2} d x d t \\
\Omega_{\alpha}^{\tau}=(0, \alpha) \times(0, \tau), \\
-\frac{\beta}{2} \int_{0}^{\tau} \Im_{t} e^{k t}\left(\frac{\partial z(\beta, t)}{\partial x}\right)^{2} d x d t \\
+\frac{\alpha}{2} \int_{0}^{\tau} \Im_{t} e^{k t}\left(\frac{\partial z(\alpha, t)}{\partial x}\right)^{2} d x d t \\
-\frac{1}{2} \int_{\Omega_{\alpha, \beta}^{\tau}} \Im_{t} e^{k t}\left(\frac{\partial z}{\partial x}\right)^{2} d x d t, \\
\Omega_{\alpha, \beta}^{\tau}=(\alpha, \beta) \times(0, \tau), \\
\frac{1}{2} \int_{0}^{\tau}\left(\beta-\frac{\beta^{2}}{2}\right) \Im_{t} e^{k t}\left(\frac{\partial z(\beta, t)}{\partial x}\right)^{2} d x d t \\
-\frac{1}{2} \int_{\Omega_{\beta}^{\tau}}(1-x) \Im_{t} e^{k t}\left(\frac{\partial z}{\partial x}\right)^{2} d x d t, \\
\Omega_{\beta}^{\tau}=(\beta, 1) \times(0, \tau) .
\end{array}\right.
\end{aligned}
$$

So, we obtain

$$
\begin{aligned}
& \int_{\Omega^{\tau}} e^{k t} z \omega d x d t \\
& =\left\{\begin{array}{c}
-\frac{1}{2} \int_{\Omega_{\alpha}^{\tau}}(1-x) \Im_{t} e^{k t}\left(\frac{\partial z}{\partial x}\right)^{2} d x d t, \\
\Omega_{\alpha}^{\tau}=(0, \alpha) \times(0, \tau), \\
-\frac{1}{2} \int_{\Omega_{\alpha, \beta}^{\tau}} \mathfrak{\Im}_{t} e^{k t}\left(\frac{\partial z}{\partial x}\right)^{2} d x d t, \\
\Omega_{\alpha, \beta}^{\tau}=(\alpha, \beta) \times(0, \tau), \\
-\frac{1}{2} \int_{\Omega_{\beta}^{\tau}}(1-x) \Im_{t} e^{k t}\left(\frac{\partial z}{\partial x}\right)^{2} d x d t, \\
\Omega_{\beta}^{\tau}=(\beta, 1) \times(0, \tau) .
\end{array}\right.
\end{aligned}
$$

Now, by combining over $\Omega^{\tau}$, we get

$$
\frac{1}{2} \int_{\Omega_{\alpha}^{\tau}}(1-x) e^{k t} z^{2} d x d t+\frac{1}{2} \int_{\Omega_{\alpha, \beta}^{\tau}} e^{k t} z^{2} d x d t
$$$$
+\frac{1}{2} \int_{\Omega_{\beta}^{\tau}}(1-x) e^{k t} z^{2} d x d t
$$$$
=\frac{\alpha^{2}}{4} \int_{0}^{\tau} \Im_{t} e^{k t}\left(\frac{\partial z(\alpha, t)}{\partial x}\right)^{2} d x d t
$$$$
-\frac{\beta^{2}}{4} \int_{0}^{\tau} \mathfrak{\Im}_{t} e^{k t}\left(\frac{\partial z(\beta, t)}{\partial x}\right)^{2} d x d t
$$$$
-\frac{1}{2} \int_{\Omega_{\alpha}^{\tau}}(1-x) \Im_{t} e^{k t}\left(\frac{\partial z}{\partial x}\right)^{2} d x d t
$$$$
-\frac{1}{2} \int_{\Omega_{\alpha, \beta}^{\tau}} \Im_{t} e^{k t}\left(\frac{\partial z}{\partial x}\right)^{2} d x d t
$$$$
-\frac{1}{2} \int_{\Omega_{\beta}^{\tau}}(1-x) \Im_{t} e^{k t}\left(\frac{\partial z}{\partial x}\right)^{2} d x d t,
$$

by taking into accont the boundary condition (40) of the function $z$ yields

$$
\begin{aligned}
& \frac{1}{2}\left(\begin{array}{c}
\int_{\Omega_{\alpha}^{\tau}}(1-x) e^{k t} z^{2} d x d t+\int_{\Omega_{\alpha, \beta}^{\tau}} e^{k t} z^{2} d x d t \\
+\int_{\Omega_{\beta}^{\tau}}(1-x) e^{t} z^{2} d x d t
\end{array}\right) \\
& \quad=-\frac{1}{2}\left(\begin{array}{c}
\int_{\Omega_{\alpha}^{\tau}}(1-x) \Im_{t} e^{k t}\left(\frac{\partial z}{\partial x}\right)^{2} d x d t+\int_{\Omega_{\alpha, \beta}^{\tau}} \Im_{t} e^{k t}\left(\frac{\partial z}{\partial x}\right)^{2} d x d t \\
\int_{\Omega_{\beta}^{\tau}}(1-x) \Im_{t} e^{k t}\left(\frac{\partial z}{\partial x}\right)^{2} d x d t
\end{array}\right) \\
& \leq 0 .
\end{aligned}
$$


Then, we obtain

$$
\begin{gathered}
\frac{1}{2}\left(\int_{\Omega_{\alpha}^{\tau}}(1-x) e^{k t} z^{2} d x d t+\int_{\Omega_{\alpha, \beta}^{\tau}} e^{k t} z^{2} d x d t\right. \\
\left.+\int_{\Omega_{\beta}^{\tau}}(1-x) e^{k t} z^{2} d x d t\right)
\end{gathered}
$$

$\leq 0$.

And thus $z=0$ in $\Omega$, then $\omega=0$ in $\Omega$. This proves Proposition 5.

We return to the proof of Theorem 4 . We have already noted that it is sufficient to prove that the set $R(L)$ dense in F.

Suppose that for some $W=\left(\omega, \omega_{0}\right) \in R(L)^{\perp}$ and for all $u \in D(L) \equiv B$, it holds

$$
\begin{aligned}
& (L u, \omega)_{F} \\
& \quad=\int_{\Omega} \mathscr{L} u \cdot \omega d x d t+\int_{0}^{1}(x+1)\left(\frac{\partial \ell u}{\partial x}\right)\left(\frac{\partial \omega_{0}}{\partial x}\right) d x
\end{aligned}
$$

Then we must prove that $W=0$. Putting $u \in D_{0}(L)$ in (47), we have

$$
\int_{\Omega} \mathscr{L} u \cdot \omega d x d t=0, \quad u \in D_{0}(L) .
$$

Hence Proposition 5 implies that $\omega=0$. Thus (47) takes the form

$$
\int_{0}^{1}(x+1)\left(\frac{\partial \ell u}{\partial x}\right)\left(\frac{\partial \omega_{0}}{\partial x}\right) d x=0, \quad u \in D(L) .
$$

Since the range of the trace operator $\ell$ is everywhere dense in the Hilbert space $F$ with the norm

$$
\left(\int_{0}^{1}\left[(x+1)\left(\frac{\partial \ell u}{\partial x}\right)^{2}\right]\right)^{1 / 2}
$$

the equality (49) implies that $\omega_{0}=0$. Hence $W=0$ implies $(\overline{R(L)}=F)$. Therefore, the proof of Theorem 4 is complete.

\section{References}

[1] A. A. Samarskiŭ, "Some problems of the theory of differential equations," Differentsial'nye Uravneniya, vol. 16, no. 11, pp. 19251935, 1980.

[2] L. I. Kamynin, "A boundary-value problem in the theory of heat conduction with non-classical boundary conditions," Zhurnal Vychislitel'noi Matematiki i Matematicheskoi Fiziki, vol. 4, pp. 1006-1024, 1964.

[3] N. I. Ionkin, "Solution of boundary value problem in heat conduction theory with nonlocl boundary conditions," Differentsial'nye Uravneniya, vol. 13, no. 2, pp. 294-304, 1977.

[4] N. I. Yurchuk, "A mixed problem with an integral condition for some parabolic equations," Differentsial'nye Uravneniya, vol. 22, no. 12, pp. 2117-2126, 1986.
[5] N. E. Benouar and N. I. Yurchuk, "A mixed problem with an integral condition for parabolic equations with a Bessel operator," Differentsial'nye Uravneniya, vol. 27, no. 12, pp. 20942098, 1991.

[6] A. Bouziani, "Mixed problem with boundary integral conditions for a certain parabolic equation," Journal of Applied Mathematics and Stochastic Analysis, vol. 9, no. 3, pp. 323-330, 1996.

[7] A. Bouziani and N.-E. Benouar, "Problème mixte avec conditions intégrales pour une classe d'équations paraboliques," Comptes Rendus de l'Académie des Sciences I, vol. 321, no. 9, pp. 1177-1182, 1995.

[8] A. Bouziani and N.-E. Benouar, "Mixed problem with integral conditions for a third order parabolic equation," Kobe Journal of Mathematics, vol. 15, no. 1, pp. 47-58, 1998.

[9] S. Mesloub and A. Bouziani, "Mixed problem with a weighted integral condition for a parabolic equation with the Bessel operator," Journal of Applied Mathematics and Stochastic Analysis, vol. 15, no. 3, pp. 291-300, 2002.

[10] J. R. Cannon, "The solution of the heat equation subject to the specification of energy," Quarterly of Applied Mathematics, vol. 21, pp. 155-160, 1963.

[11] A. M. Nakhushev, "On a certain approximate method for boundary-value problems for differential equations and their applications in ground waters dynamics," Differentsial'nye Uravneniya, vol. 18, no. 1, pp. 72-81, 1982.

[12] V. A. Vodakhova, "A boundary value problem with A. M. Nakhushev's nonlocal condition for a pseudoparabolic equation of moisture transfer," Differentsial'nye Uravneniya, vol. 18, no. 2, pp. 280-285, 1982.

[13] A. V. Kartynnik, "Three points boundary value problern with an integral space variables conditions for second order parabolic equations," Differentsial'nye Uravneniya, vol. 26, no. 9, pp. 15681575,1990 .

[14] Y. Lin, Parabolic partial differential equation subject to nonlocal boundary conditions [Ph.D. thesis], Washington State University, Pullman, Wash, USA, 1988.

[15] N. I. Ionkin, "Stability of a problem in heat-condition," Differentsial'nye Uravneniya, vol. 13, no. 2, pp. 294-304, 1977.

[16] A. V. Kartynnik, "A three-point mixed problem with an integral condition with respect to the space variable for second-order parabolic equations," Differentsial'nye Uravneniya, vol. 26, no. 9, pp. 1568-1575, 1990.

[17] N. I. Kamynin, "A boundary value problem in the theory of the condition with non classical boundary condition," Zhurnal Vychislitel'noi Matematiki i Matematicheskoi Fiziki, vol. 43, no. 6, pp. 1006-1024, 1964.

[18] A. L. Marhoune, "A three-point boundary value problem with an integral two-space-variables condition for parabolic equations," Computers \& Mathematics with Applications, vol. 53, no. 6, pp. 940-947, 2007.

[19] A. L. Marhoune and F. Lakhal, "An integral two space-variables condition for parabolic equations," Journal of Mathematics and Statistics, vol. 8, no. 2, pp. 185-190.

[20] B. Cahlon, D. M. Kulkarni, and P. Shi, "Stepwise stability for the heat equation with a nonlocal constraint," SIAM Journal on Numerical Analysis, vol. 32, no. 2, pp. 571-593, 1995. 


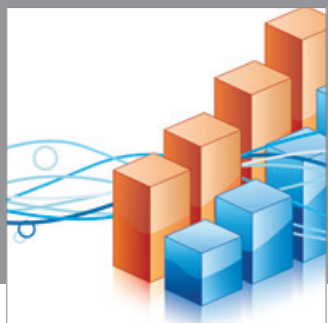

Advances in

Operations Research

mansans

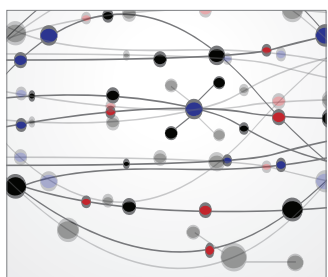

The Scientific World Journal
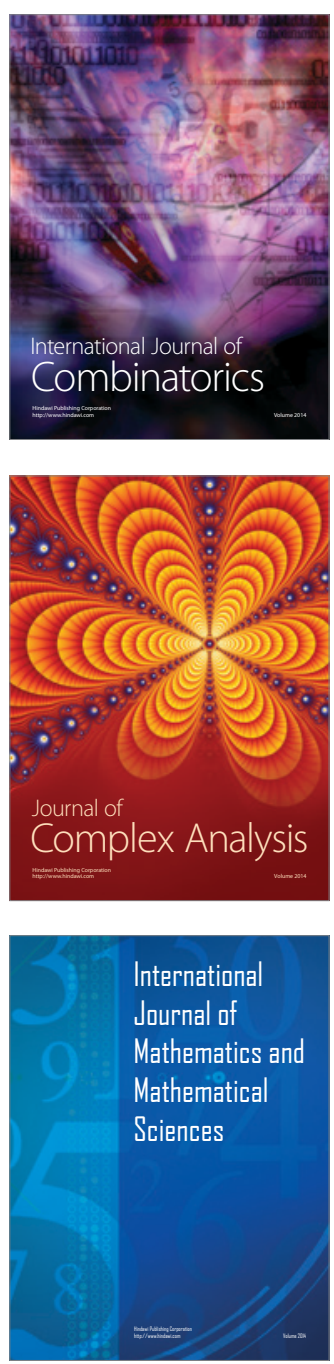
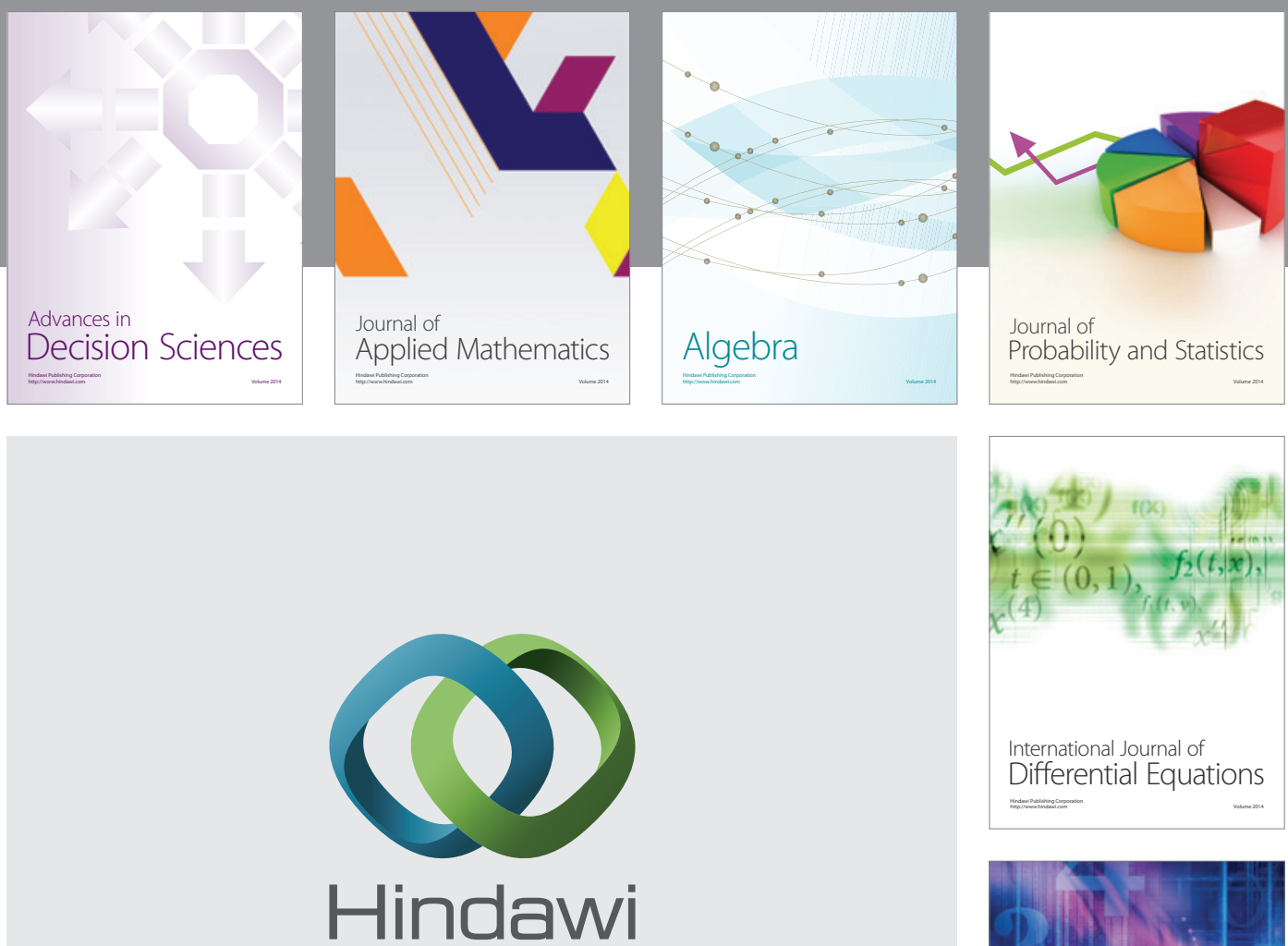

Submit your manuscripts at http://www.hindawi.com
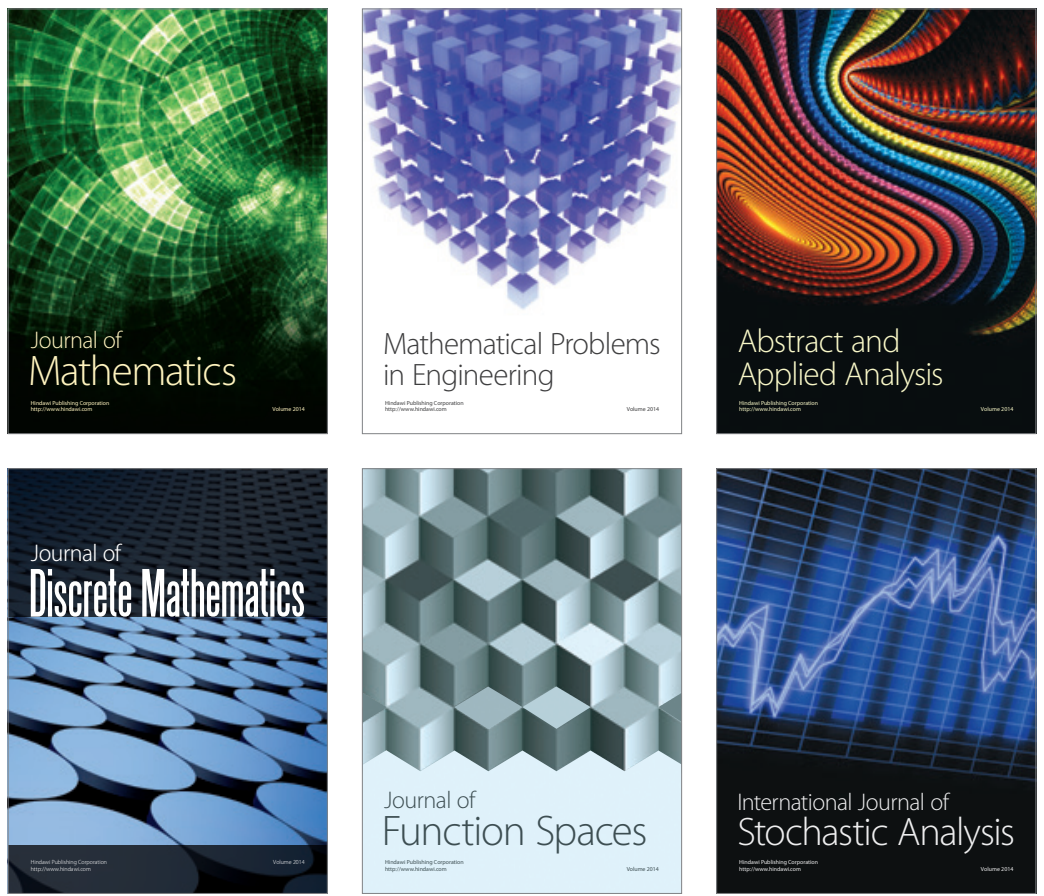

Journal of

Function Spaces

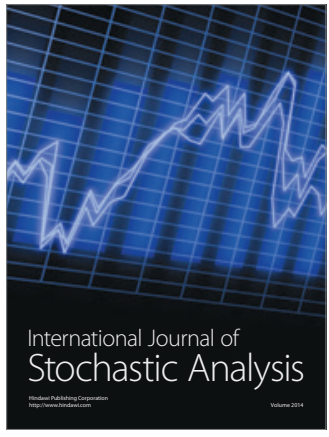

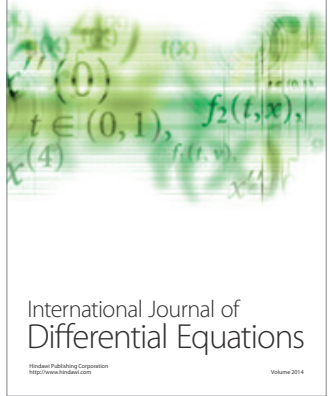
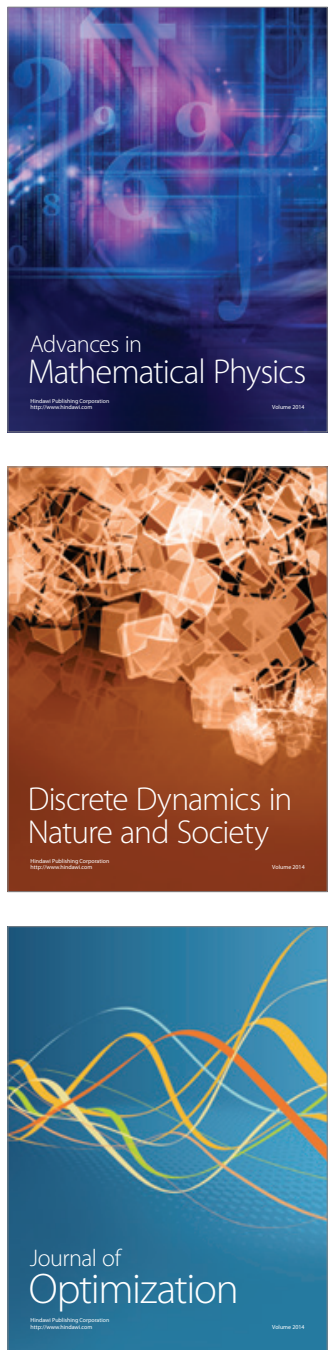